\title{
VELOCITY MODEL BUILDING FOR 3D PRE-STACK DEPTH MIGRATION - A CASE STUDY
}

DAVE HINKLEY, RACHEL HO and STAN LEE Mobil E\&P Technical Center, PO Box 650232, Dallas TX 75265-0232, USA

\section{Summary}

In this case study we used three different velocity estimation methods, SIVA/RAYMAP (Sierra ${ }^{\mathrm{TM}}$ ) velocity analysis package, focusing analysis, and residual velocity analysis, to build velocity models for $3 \mathrm{D}$ prestack depth migration. The first two methods are more interpretation oriented than the last one. The imaging results from 3D Kirchhoff prestack depth migration from these three different models are discussed in this paper.

\section{Imaging Problem}

The area covered is $10 \mathrm{~km}$ by $5 \mathrm{~km}$. There is no lateral velocity change between sea level and $2 \mathrm{~km}$ in depth, just a smoothly increasing with depth (i.e., $\mathrm{V}=\mathrm{V}_{0}+\mathrm{kZ}$ ). Below $2 \mathrm{~km}$, a low velocity high pressured zone causes strong lateral velocity variations. Synthetic calculation has shown that the conventional time migration causes a horizontal positioning error up to 210 meters and a vertical error up to 140 meters due to the strong lateral velocity variations. In addition, the top of this low velocity zone has an irregular morphology. Out-of-plane events are then presented in the $2 \mathrm{D}$ seismic line. The conventional 3D DMO followed by 3D poststack time migration failed to image below 3 seconds( Figure 1-a) in the area of interest. Conventional time migration produced sweeping noise that the deeper reflections were masked. Therefore, a full 3D prestack depth migration was considered to resolve this complex imaging problem.

\section{D Velocity Model Building}

The first method was based on the SIVA/RAYMAP(Sierra ${ }^{\mathrm{TM}}$ ) package. This package uses CMP gathers to estimate the dip and corrected interval velocity and performs a map migration to produce a time-depth conversion. The velocity model is built interactively from the top layer to the bottom layer. Forty two seismic profiles were used for the velocity estimation. Velocity analysis was performed every 250 meters along the in-line direction and 500 meters along the cross-line direction. Velocities were estimated at a total of 1481 CMP locations.

The second method employed a Kirchhoff based prestack focusing analysis to determine interval velocity. The focus panels were interpreted using Mobil's proprietary velocity building package. Focusing analysis was performed every 200 meters in the in-line direction, and every 250 meters in the cross-line direction. 
The third method used a residual velocity analysis to build a velocity model. The data were DMO corrected, time migrated in common offset profiles, and then regathered to CDP locations. The stacking velocities were estimated in the CDP locations. Finally, a constrained inversion to interval velocities was performed. Residual velocity analysis was conducted every 75 meters along the inline direction, and every 250 meters along the cross-line direction.

\section{D Prestack Depth Migration}

After the velocity models were built, the three velocity models were interpolated into finer grids for travel time calculation using finite difference eikonal equation. The travel times calculated were used as the input to 3D Kirchhoff prestack depth migration. Both travel time and migration computations were performed on a CM-5 massively parallel computer. The imaging results from the three velocity models are shown in Figure 1-b,c,d.

Compare the imaging results from the conventional 3D DMO followed by poststack time migration( Figure 1-a) with those from 3D prestack depth migration (Figures 1-b,c,d ), the latter show significant improvement in the imaging quality below $2 \mathrm{~km}$. The fault definition is much clear using 3D prestack depth migration.

The 3D prestack depth migration results using the three models were very similar. This may due to the smoothness of the models and the fact that the velocity has much less complexity along the cross-line direction. There were two reasons for smoothing the velocity models: (1) Velocity analysis on the 3D data volume is an expensive and time consuming process. Therefore, the velocity analysis locations were sparse. (2) The smoothness of the velocity model helped the 3D prestack depth migration processing by avoiding instabilities associated with strong velocity boundaries and by speeding up the travel times calculation.

\section{Acknowledgement}

The authors gratefully acknowledge Kevin Hellman, Stew Levin, and Jorge Pita for conducting the 3D prestack depth migration. We also thank to our producing affiliate to support the study. We are grateful to the management of Mobil for permission to publish this paper.

\section{Reference}

SIVA and RAYMAP, Sierra ${ }^{\mathrm{TM}}$, User manual, 1988 


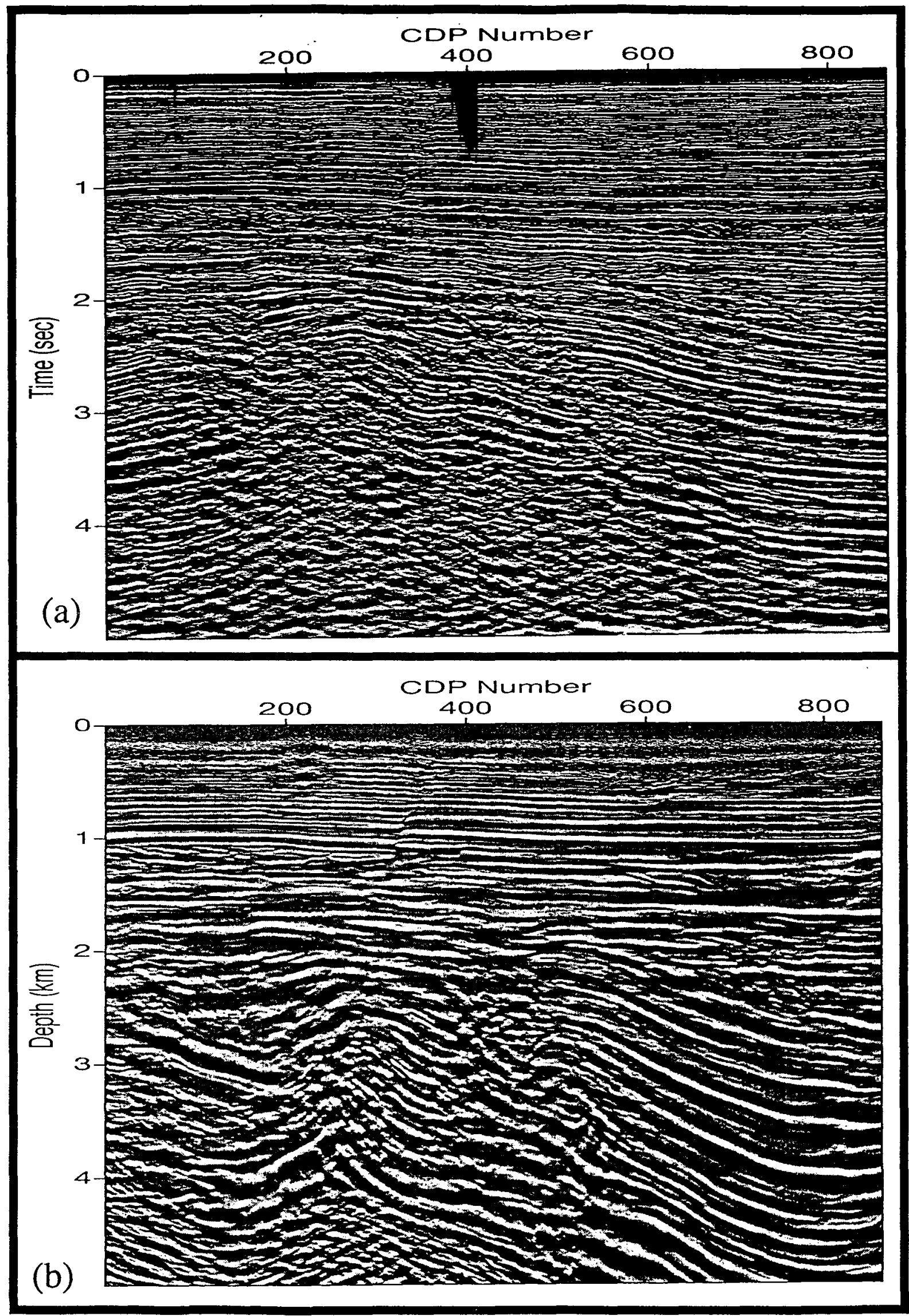

Figure 1. (a) 3D DMO followed by 3D poststack time migration

(b) 3D prestack depth migration using model a 


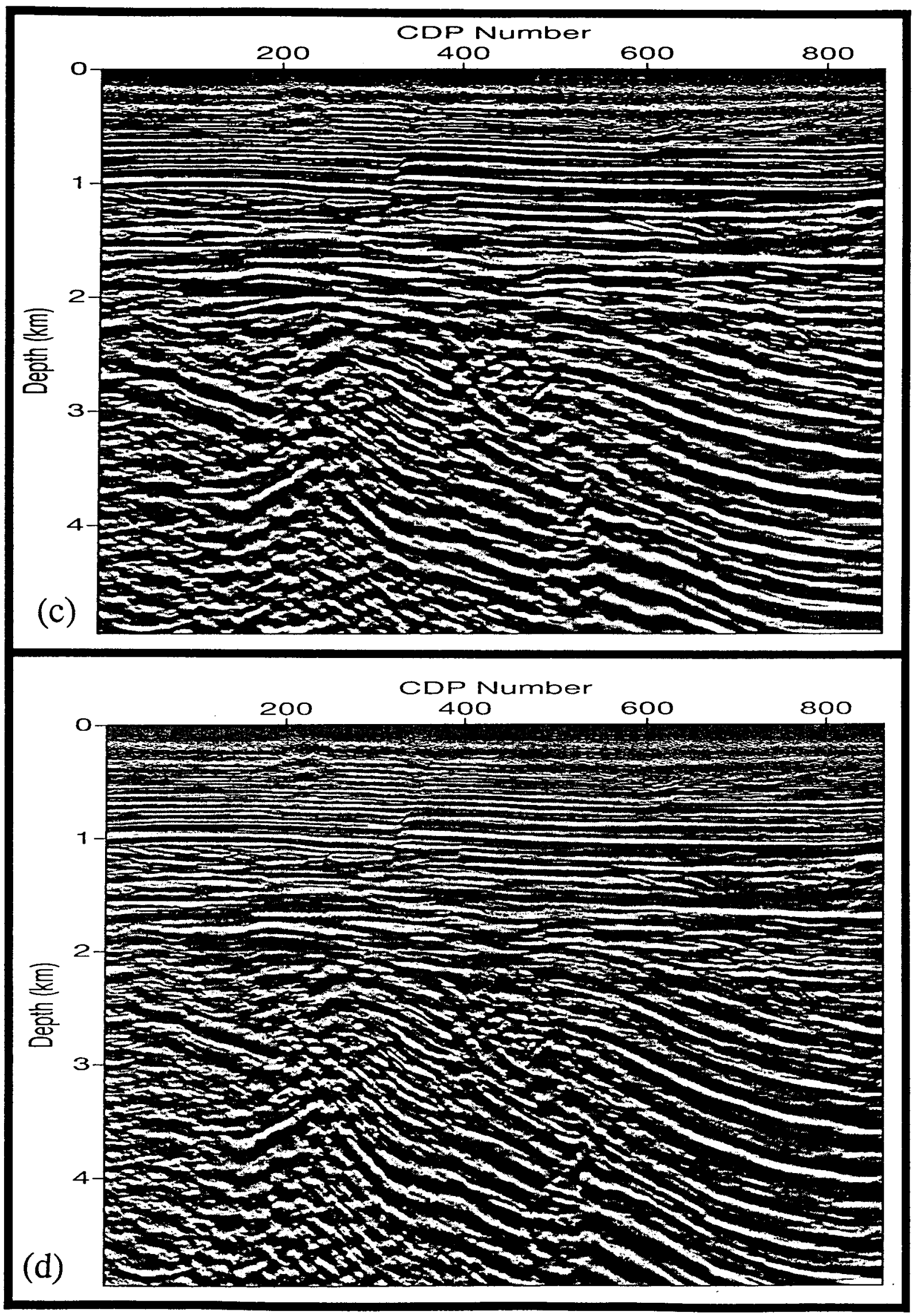

Figure 1. (c) 3D prestack depth migration using model b (d) 3D prestack depth migration using model $\mathrm{c}$ 